\title{
Spin-Dependent Tunneling in a Magnetic Field for Junctions Involving Normal and Superconducting CDW Metals
}

\author{
T. Ekino ${ }^{a}$, A.M. Gabovich ${ }^{b}$, Mai SuAn Li $^{c}$, M. Pf̧KalA $^{d}$, \\ H. SZYMCZAK ${ }^{c}$ AND A.I. VOITENKO ${ }^{b}$ \\ ${ }^{a}$ Faculty of Integrated Arts and Sciences, Hiroshima University \\ Higashi-Hiroshima 7398521, Japan \\ ${ }^{b}$ Institute of Physics, prospekt Nauki 46, Kiev, Ukraine \\ ${ }^{c}$ Institute of Physics, Polish Academy of Sciences \\ al. Lotników 32/46, 02-668 Warsaw, Poland \\ ${ }^{d}$ Department of Chemistry, Warsaw University \\ Żwirki i Wigury 101, Warsaw, Poland
}

\begin{abstract}
The voltage, $V$, dependences of the differential tunnel conductance $G(V)$ were calculated for two kinds of junctions involving normal and superconducting charge-density-wave metals (CDWMs) in the external magnetic field $H$. The first is a non-symmetrical junction with a CDWM electrode being biased with respect to a ferromagnet. Calculations show that the paramagnetic splitting occurs between spin-up and spin-down components of $G(V)$, similar to what is observed when a superconducting electrode is used instead of the CDWM one. The second setup is symmetric (CDWMI-CDWM), where I denotes an insulator. If at least one of the CDWM electrodes is normal and $H \neq 0, G(V)$ will also be spin-split.
\end{abstract}

PACS numbers: 71.45.Lr, 74.50.+r, 85.75.-d

\section{Introduction}

Spintronics is a new highly productive branch of electronics. Among various spintronics applications, there is an older one invented by Tedrow and Meservey [1]. They predicted that the gapped character of the quasiparticle density of states (DOS) in the Bardeen-Cooper-Schrieffer (BCS) superconductors allows one to measure the spin-polarization $P$ of the tunnel current across an FM-I-S junction, where FM, I, and S denote a ferromagnet, an insulator, and a superconductor, respectively, if the junction is in an external magnetic field $H$ [1]. The method 
has, nevertheless, several drawbacks. These are spin-orbit scattering and the Meissner orbital effect for superconductors.

At the same time, there exists a class of solid-state objects, namely, charge-density-wave (CDW) insulators or metals (CDWMs) with paramagnetic properties very similar to those of superconductors but possessing energy gaps induced by electron-hole rather than Cooper pairing $[2,3]$. Therefore, we propose normal and superconducting CDWMs as polarization tunnel detectors, having certain advantages over conventional superconductors.

\section{Formulation}

\subsection{Some basic properties of charge-density-wave metals}

The Bilbro-McMillan Hamiltonian of the partially-dielectrized superconducting CDW metal [4] is our starting point. According to the model [4] (see also reviews $[5,6]$ ), the Fermi state (FS) of the CDWM is split into two degenerate (nested, $d, i=1,2$ ) and one non-degenerate (non-nested, $n, i=3$ ) sections. For the former, the bare quasiparticle spectrum branches $\xi_{1,2}(\boldsymbol{p})$ reckoned from the common Fermi level are linked by the relation $\xi_{1}(\boldsymbol{p})=-\xi_{2}(\boldsymbol{p}+\boldsymbol{Q})$, where $\boldsymbol{Q}$ is the CDW vector. Due to the interaction between quasiparticles from different $d$ FS sections, a pairing-like correlation arises and a dielectric CDW order parameter $\tilde{\Sigma} \equiv \Sigma \mathrm{e}^{\mathrm{i} \varphi}$ emerges at both nested sections. The rest of the FS remains undistorted by CDWs and is described by the $n$ electron spectrum branch $\xi_{3}(\boldsymbol{p})$. The portion of the FS gapped by the CDW instability is described by a dielectric-gapping (dielectrization) parameter $\mu=N_{d 0}(0) / N_{0}(0)$, where $N_{0}(0)=N_{n 0}(0)+N_{d 0}(0)$ is the total initial (above $T_{d}$ ) electronic DOS on the FS, and $N_{d 0}(0)$ and $N_{n 0}(0)$ are the relevant DOSes on the $d$ and $n$ FS sections, respectively.

At the critical temperature of the superconducting transition $T_{\mathrm{c}}<T_{d}$, a superconducting order parameter $\widetilde{\Delta} \equiv \Delta \mathrm{e}^{\mathrm{i} \varphi}$ emerges, unique for the whole FS. It means that below $T_{\mathrm{c}}$ the $d \mathrm{FS}$ sections are distorted by two kinds of interactions, which leads to a combined energy gap, whereas the electron spectrum on the $n$ section exhibits a gap $\Delta[4-6]$. One should stress that the phase $\varphi$ of the superconducting order parameter does not reveal itself for the quasiparticle current $J$ studied in the present work, although it is well known that this quantity controls the coherent Josephson current through a weak link between superconductors.

CDWs may be commensurate or incommensurate with the background crystal lattice. In the excitonic insulator model, the Coulomb-induced distortion below $T_{d}$ is commensurate. Moreover, the phase of the order parameter in excitonic insulators is always pinned, and $\tilde{\Sigma}$ is either a positive or a negative quantity $[5,7,8]$. On the other hand, in the Peierls insulators, incommensurate CDWs may exhibit a rich dynamics, although in the actual current measurements they may be pinned with an arbitrarily frozen phase $\varphi[9]$.

At $H \neq 0$, the quasiparticle currents in junctions involving CDWMs are governed by the temporal Green functions $\mathcal{G}_{i j}(\omega)$ describing the electrodes [10], where the subscripts $i, j=1,2,3$ label the FS sections: $\mathcal{G}_{11}=\mathcal{G}_{22} \equiv \mathcal{G}_{d}, \mathcal{G}_{12}=$ 
$\mathcal{G}_{21} \equiv \mathcal{G}_{\mathrm{c}}, \mathcal{G}_{33} \equiv \mathcal{G}_{n}$. For all the other $i j$-combinations, $\mathcal{G}_{i j}=0$. The function $\mathcal{G}_{\mathrm{c}}$ describes electron-hole pairing.

As is well known, the voltage dependence of $J$ is exponential for large and ohmic for small $V$. We shall not extend the subsequent analysis beyond the ohmic regime, since experimentally relevant superconducting and dielectric gap energies fall within the range $0.2 \mathrm{meV}<\Sigma<30 \mathrm{meV}$, whereas the deviations from the $\mathrm{Ohm}$ law become important for $\mathrm{eV}$ comparable to the conduction band width $W \geq 1 \mathrm{eV}$ for either of the electrodes. (Hereafter, $e>0$ is the elementary charge.)

In studying tunnel currents between CDWMs, the standard tunnel Hamiltonian method will be used. One can introduce a unique parameter $R$ representing the junction resistance in the normal state. The quantity $R$ is reciprocal to the averaged square of the tunnel matrix elements $[5,10]$. If $H$ (the spatial $z$ axis is chosen to be aligned with $\boldsymbol{H}$ ) is switched on, the $d$ and $n$ states exhibit quite different paramagnetic behavior.

Let us describe the paramagnetic properties of CDWMs at non-zero $H<H_{p}^{\mathrm{CDWM}}$. Electrons with the spin projection $s_{z}=+1 / 2$ on $\boldsymbol{H}$ increase their energies by $\mu_{\mathrm{B}}^{*} \mathrm{H}$, while the electrons with the opposite spin direction, $s_{z}=-1 / 2$, reduce their energies by the same amount. Here, $\mu_{\mathrm{B}}{ }^{*}=e \hbar /\left(2 m^{*} c\right)$ is the effective Bohr magneton, $c$ is the velocity of light, and $m^{*}$ is the effective mass of current carriers. Henceforth, quasiparticles with either spin direction will be labeled by "+" or "-", respectively.

Quasiparticles belonging to the $n$ section behave in a conventional manner inherent to normal metals. Namely, the chemical potential $\widetilde{\mu}$ shifts by a negligible value of the order of $\left(\mu_{B}^{*} H / E_{\mathrm{F}}\right)^{2} \ll 1$. It is necessary to remind that in itinerant Stoner ferromagnets this is not the case, and $\widetilde{\mu}$ is altered conspicuously by the respective spin polarization.

The paramagnetic splitting of quasiparticle states from the gapped FS ( $n$ section below $T_{\mathrm{c}}$ and $d$ one below $T_{d}$ ) can be examined analogously to that for superconductors [1]. In what follows, we shall describe tunneling in the magnetic field $H \neq 0$ with the help of the temporal Green functions $\mathcal{G}_{d}, \mathcal{G}_{c}$, and $\mathcal{G}_{n}$ mentioned above. The only modification, in comparison to the expressions of Refs. [5, 10], is that now the number of the Green functions is doubled: six relevant $H$-dependent functions $\mathcal{G}_{d s}, \mathcal{G}_{c s}$, and $\mathcal{G}_{n s}$ are denoted by an extra subscript $s= \pm$. They are functions of the relevant variables $\omega \mp \mu_{\mathrm{B}}^{*} H$, whose signs are inverse to those of $s$.

\subsection{Current-voltage characteristics}

\subsubsection{Non-symmetrical junctions}

While examining current-voltage characteristics (CVCs) for non-symmetrical junctions, the bias voltage $V$ is chosen as the difference between the electric potentials of the itinerant (Stoner) FM and the CDWM (normal or superconducting): $V \equiv V_{\mathrm{FM}}-V_{\mathrm{CDWM}}$.

It is presumed that all domains inside the ferromagnet are completely aligned along the field direction [1]. We also anticipate that the initial bulk $P$ is preserved 
during tunneling. We fully recognize that, generally speaking, this is not the case, the boundary and disorder effects being important [11].

Making use of the BCS function $\tilde{\Sigma}(T)$ and following the method developed for conventional superconductors [12], we calculate $J(V)$ between the FM and the CDWM. Then, the overall tunnel current $J$ can be described as consisting of different terms $J_{i \mp}$ corresponding to various combinations of spin-dependent temporal Green's functions. The upper (lower) sign corresponds to the "majority" ("minority") spin orientation, respectively. In the basic approach adopted here, there is no problem with $\mathcal{G}_{\mp}^{\mathrm{FM}}(\omega)$. At the same time, there emerge six quantities $\mathcal{G}_{i \mp}^{\mathrm{CDWM}}(\omega)$ instead of a unique spin-projection pair $\mathcal{G}_{\mp}^{\mathrm{BCS}}(\omega)$ specifying the BCS state.

After the straightforward procedure, we obtain

$$
J(V)=\sum_{f=n, d, \mathrm{c} ; s=-,+} J_{f s}(V) .
$$

One should bear in mind that the current components depend on the phase $\varphi$ of $\tilde{\Sigma}=\Sigma \mathrm{e}^{\mathrm{i} \varphi}$, whereas the thermodynamic properties of CDW superconductors are degenerate with respect to $s$ [13]. We suggested that quasiparticles originating from each FS section make their contributions to the total current proportional to the DOS of this section. This means an absence of the directional tunneling, which is possible, in principle.

The difference between the problem in point and its counterpart appropriate to "pure" superconductivity is the emergence of the terms $J_{\mathrm{c} \mp}$. They originate from the interband Green function [7]. The appearance of the terms $J_{\text {c干 }}$ leads to the drastic asymmetry of the CVCs of non-symmetrical tunnel junctions involving CDWMs [7], as opposed to symmetrical CVC for similar non-symmetrical junctions based on conventional superconductors. In incommensurate CDWMs, the order parameter phase $\varphi$ is arbitrary [9]. For commensurate CDWs, $\varphi$ is either 0 or $\pi$. Then, relevant equations describe tunneling between, e.g., excitonic insulators $[8,14]$, for which the phase is pinned by the inter-band four-fermion interaction. In principle, another situation is also possible, when a tunnel contact area is large enough to span several zones with the varying $\varphi$. As a consequence, the term $J_{\text {cF }}$ would be averaged to some extent and the CVC asymmetry would be substantially reduced.

\subsubsection{Symmetrical junctions}

For a junction between dissimilar CDWMs (a CDWM'-I-CDWM junction) in the magnetic field $H$, the $G(V)$-peak structure is more involved if both the CDWMs are normal than if they are superconducting. Such an amazing situation occurs because the spin splitting is absent if the whole FS of each electrode is gapped whatever the nature of the collective energy gap (gaps). As was indicated above, this was first recognized for BCS $s$-wave superconductors [1]. Thus, in our case, the spin splitting would emerge only if the dielectric gapping is partial and $\Delta=0$. Here, we are going to examine major features of a simple case of symmetrical junctions with identical normal or superconducting CDWM electrodes. 
In the adopted model [4], the symmetry means the equalities $\Sigma=\Sigma^{\prime}$ and $\mu=\mu^{\prime}$, although the phases $\varphi^{\prime}$ and $\varphi$ may differ for states with the same energy.

\section{Numerical results}

Below, all the results are expressed as the dependences of the dimensionless conductance $R \mathrm{~d} J / \mathrm{d} V$ of the FM-I-CDWM and CDWM-I-CDWM junctions on the dimensionless bias voltage $\mathrm{eV} / \Sigma_{0}$, where $\Sigma_{0} \equiv \Sigma(T=0)$. The dimensionless parameters of the problem are the reduced external magnetic field $h=\mu_{\mathrm{B}}^{*} H / \Sigma_{0}$, the reduced temperature $t=T / \Sigma_{0}, T$ is the temperature, the Boltzmann constant $k_{\mathrm{B}}=1$, and the polarization $P$.

\subsection{Non-symmetrical junctions}

The $G(V)$, split in the external magnetic field, is highly asymmetrical at $\varphi=0$ for the FM-I-normal CDWM junction, contrary to the symmetrical patterns appropriate to tunnel junctions involving conventional superconductors, no matter whether those junctions are symmetrical or not. Mathematically, it stems from an almost total compensation between $G_{d}(V)$ - and $G_{\mathrm{c}}(V)$-peculiarities at voltages of one sign and their enhancement at voltages of the other sign. If $H$ is switched on, the electronic DOS peak splits as in the case of superconductors [1]. The spin splitting crucially depends on $P$, but is noticeable only for one CVC branch ( $V>0$ in the case $\varphi=0$, the other branch contains only remnants of the gap-related features).

Such dependences are depicted in Fig. 1 for fixed $h$ and $P$, but for various phases of the CDW order parameter. It is clear from the figure that the CDW phase plays a crucial role in the determination of the CVC forms. If a spot generating the tunneling current includes a set of micro-tips with random phase distribution, the terms $J_{\mathrm{c} \mp}$ are averaged out, so this case corresponds to the curve labeled by $\varphi=\pi / 2$. Another interesting feature is the violation of the generalized symmetry

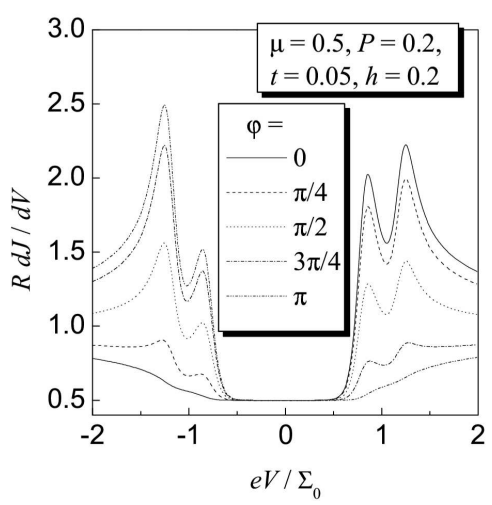

Fig. 1. Voltage, $V$, dependences of the dimensionless conductance $R \mathrm{~d} J / \mathrm{d} V=R G(V)$ in a tunnel junction between FM and normal CDWM electrode embedded into the magnetic field $H$ for various phases of the CDW order parameters. 
relationships $J(V,-\Sigma, P=0)=-J(-V, \Sigma, P=0)$ and $G(V,-\Sigma, P=0)=$ $G(-V, \Sigma, P=0)$ obtained earlier [10].

The simultaneous DOS distortion by order parameters $\Delta$ and $\tilde{\Sigma}$ leads to an interplay between constituents of $G(V)$. This is shown in Fig. 2 when CDWM is a superconductor, $\varphi=0$, and the ratio $\delta_{0} \equiv \Delta_{0} / \Sigma_{0}=0.5$. Here, $\Delta_{0}$ is the value of the superconducting gap in the hypothetical case where CDWs are switched off $\left(T_{d}=\Sigma=0\right)$. Let us note that $H$ is smaller here than the paramagnetic limit for CDW superconductors [15]. One sees that both the square-root singularities are split. Only the $\Delta$-driven peaks of $G(V)$ appear in the negative-bias branch.

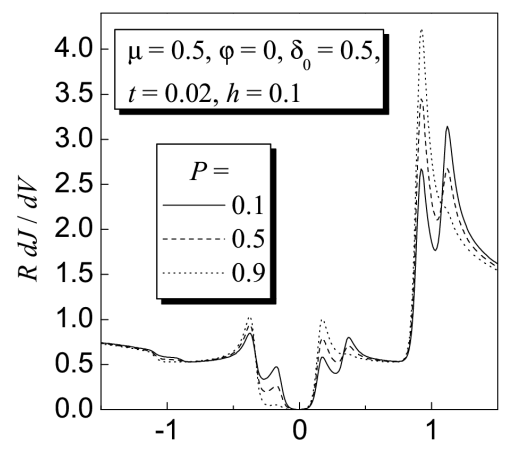

Fig. 2

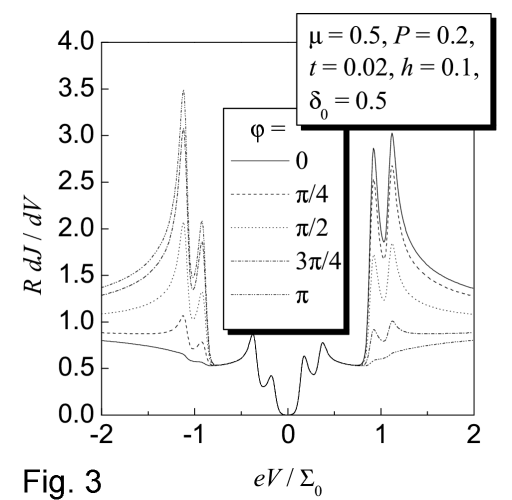

Fig. 3

Fig. 2. The dependences $G(V)$ at various $P$ for a superconducting CDWM.

Fig. 3. The same as in Fig. 1 but for a superconducting CDWM.

The dependences of CVCs of a FM-I-superconducting CDWM junction are shown in Fig. 3 for various $\varphi$. One sees that, similarly to the pattern shown in Fig. 1, the CDW-driven singularities are being "transferred" from the positive- $V$ branch to the negative one with increasing $\varphi$.

It would be challenging to seek for the synchronous spin splitting of pseudogap- and superconducting-gap-induced features in CVCs of junctions involving high- $T_{\mathrm{c}}$ cuprates, similar to those shown in Figs. 2 and 3. Such a search might be of help in a long-term struggle to understand the pseudogap nature $[5,6]$.

\subsection{Symmetrical junctions}

In Fig. 4 the CVCs for a symmetrical normal CDWM-I-normal CDWM tunnel junction are shown for various values of the order parameter phase for the right electrode $\varphi_{r}\left(\varphi_{l}=0\right.$ is the phase of the left electrode). The CVCs with $\varphi_{r}=0$ or $\pi$ correspond to the excitonic insulator state. At the same time, $\varphi_{r}=\pi / 2$ describes the intermediate situation and is also appropriate to the case when the tunnel current is collected from several one-dimensional chains of the Peierls insulator, so that the phase-dependent contribution to $J$ is averaged out. The peaks of $G(V)$ in Fig. 4 are split due to the paramagnetic effect. They correspond to the transition of quasiparticles from the $d$ FS sections from, e.g., the 


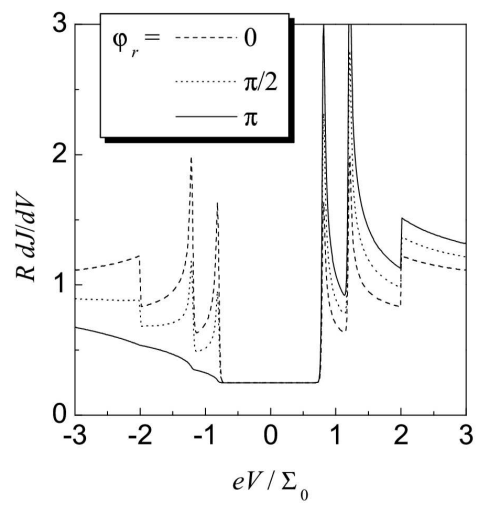

Fig. 4. The same as in Fig. 1 for a junction between two thermodynamically equivalent normal CDWM electrodes. Here $h=0.2, t=0.01, \mu=0.5$. The phase of the CDW order parameter for the left electrode $\varphi_{l}=0$.

left electrode and the $n$ section from the right one and vice versa. This splitting preserves for arbitrary phases of $\tilde{\Sigma}$. The step-like peculiarities, which describe inter-electrode tunnel transitions between the $d$ FS sections, remain unsplit.

\section{Conclusions}

Thus, we have predicted a new type of the tunnel junction, where paramagnetic splitting of the differential conductivity peaks is to appear. The variety of tunnel processes may be very rich, because the current components depend on the CDW phases in these electrodes. If one electrode is ferromagnetic, its polarization $P$ should influence the resulting $J(V)$ and $G(V)$ similarly to sandwiches involving superconductors [1].

We propose CDW metals $\alpha$-(ET) $)_{2} \mathrm{MHg}(\mathrm{SCN})_{4}(\mathrm{M}=\mathrm{K}, \mathrm{Tl}, \mathrm{Rb})[16]$ and $\operatorname{Per}_{2}\left[\mathrm{M}(\mathrm{mnt})_{2}\right](\mathrm{M}=\mathrm{Au}, \mathrm{Pt})[3]$ as possible candidates for the CDW partner of FMs in tunnel sandwiches. The predicted $G(V)$ splitting induced by the paramagnetic action of the magnetic field $H$ can be observed for any CDW metal.

An appearance of superconductivity for smaller $T<T_{\mathrm{c}}<T_{d}$ in any specific CDW substance (see reviews $[5,6]$ ) may serve as a clear indication that this material is a metal rather than an insulator, and, therefore, of its ability to demonstrate the Zeeman spin splitting. Hence, the low-dimensional metals exhibiting CDW instabilities, such as $\mathrm{NbSe}_{3}, \mathrm{Nb}_{3} \mathrm{Te}_{4}, \mathrm{Li}_{0.9} \mathrm{Mo}_{6} \mathrm{O}_{17}, \mathrm{Tl}_{2} \mathrm{Mo}_{6} \mathrm{Se}_{6}$, layered dichalcogenides, alloys with A15 and $\mathrm{C} 15$ structures, $\mathrm{Lu}_{5} \mathrm{Ir}_{4} \mathrm{Si}_{10}, \mathrm{P}_{4} \mathrm{~W}_{14} \mathrm{O}_{50}$, alkali-metal-doped tungsten bronzes, and solid solutions $\mathrm{BaPb}_{1-x} \mathrm{Bi}_{x} \mathrm{O}_{3}$ may serve as good candidates. Superconducting cuprates can be suggested as another class of substances, where the CDW-triggered spin splitting in the magnetic field can be observed. 


\section{Acknowledgments}

A.M.G. and A.I.V. are grateful to Kasa im. J. Mianowskiego (Mianowski Foundation) and Fundacja na Rzecz Nauki Polskiej (Foundation for Polish Science) for supporting their visits to Warsaw University and the Institute of Physics of the Polish Academy of Sciences. M.S.L. acknowledges the support of the State Committee for Scientific Research (Poland) grant No. 1P03B01827. The research was partly supported by the NATO grant PST.CLG.979446.

\section{References}

[1] R. Meservey, P.M. Tedrow, Phys. Rep. 238, 173 (1994).

[2] R.H. McKenzie, cond-mat/9706235.

[3] D. Graf, J.S. Brooks, E.S. Choi, S. Uji, J.C. Dias, M. Almeida, M. Matos, Phys. Rev. B 69, 125113 (2004).

[4] G. Bilbro, W.L. McMillan, Phys. Rev. B 14, 1887 (1976).

[5] A.M. Gabovich, A.I. Voitenko, M. Ausloos, Phys. Rep. 367, 583 (2002).

[6] A.M. Gabovich, A.I. Voitenko, J.F. Annett, M. Ausloos, Supercond. Sci. Technol. 14, R1 (2001).

[7] A.M. Gabovich, A.I. Voitenko, Phys. Rev. B 56, 7785 (1997).

[8] Yu.V. Kopaev, Trudy Fiz. Inst. Akad. Nauk SSSR 86, 3 (1975).

[9] G. Grüner, Density Waves in Solids, Addison-Wesley, Reading, Massachusetts 1994.

[10] A.M. Gabovich, A.I. Voitenko, Phys. Rev. B 52, 7437 (1995).

[11] E.Yu. Tsymbal, K.D. Belashchenko, J. Appl. Phys. 97, 10 C910 (2005).

[12] A.I. Larkin, Yu.N. Ovchinnikov, Zh. Eksp. Teor. Fiz. 51, 1535 (1966).

[13] A.M. Gabovich, A.S. Gerber, A.S. Shpigel, Phys. Status Solidi B 141, 575 (1987).

[14] B.I. Halperin, T.M. Rice, Solid State Phys. 21, 115 (1968).

[15] A.M. Gabovich, A.I. Voitenko, T. Ekino, J. Phys., Condens. Matter 16, 3681 (2004)

[16] J. Singleton, Rep. Prog. Phys. 63, 1111 (2000). 